\begin{tabular}{ccc}
\hline International Journal of Engineering \& Technology, $7(3.36)(2018) 118-120$ \\
SPC \\
Website w ww. sciencepubco.com/index.php/IJET \\
Research paper
\end{tabular}

\title{
Vibro-Acoustics for Reli-ability Modeling of Un-derwater Pipeline using Wireless Sensor Network (WSN), with Minimum Energy Consumption
}

\author{
Yusuf Saleh $^{1 *}$, Muhammad Sani Yahya ${ }^{2}$, Aliyu Bukar Dala ${ }^{3}$ \\ ${ }^{12}$ Department of Electrical and Electronics Engineering, Abubakar Tafawa Balewa University Bauchi \\ ${ }^{3}$ Department of Computer and Communication Engineering, Abubakar Tafawa Balewa University Bauchi \\ *Corresponding author E-mail: ysaleh@atbu.edu.ng
}

\begin{abstract}
WSN is a network of clusters of sensors nodes which sense the parameter and communicates to a server. Target is to develop underwater pipeline monitoring system remotely with lower energy consumption. Reliability maintenance will effectively give optimum performance of pipeline system operation. Reliability ensures optimum performance in the range of operation based on the behavior of the system. Validation of the mathematical model for the flow in pipeline system is used to study vibrations as elements that affect the reliability of the pipeline. In this, the focus is to validate and model the reliability for the pipeline system from the vibrations for predictive maintenance and optimum performance. Subsequently, vibro-acoustics will be used to model the reliability equation. A software can be developed for simulating the reliability model for pipeline system behavior from the vibration factors and use for predictive maintenance. Reliability factors of mean time to failure, repair and others will be put in to use to develop the original model for the pipeline. A hypothesis of $90 \%$ predictive model from the reliability under pipeline system behavior will be developed.
\end{abstract}

Keywords: maintenance; model; performance; predictive; reliability;; vibrations; vibro-acoustics; WSN.

\section{Introduction}

WSN is a cluster which contains sensor nodes with one or more sensors attached and communicate with each other and route data. One of the sensor node technologies provided by Crossbow Technology Company is Mica2 Mote. The node is required to consume low energy due to limited supply (battery power supply at remote location) with several sensors attached to the node. WSN has resource constrain in the hardware (power, memory and others) due to size and peculiar use in remote location[1]. The research focus on reliability model for only pipelines and use of WSN under water with low power usage. Acoustic sensing was used as a detection technique for the sound of insects [2]. This can be developed to serve for pipeline monitoring underwater with lower energy consumption through routing protocol (Threshold Sensitive Energy Efficient Sensor Network Protocol-TEEN, Adaptive Periodic Threshold-Sensitive Energy Efficient Sensor NetworkAPTEEN and Low Energy Adaptive Clustering HierarchyLEACH) in WSN and user-friendly interface (LabVIEW- Laboratory Virtual Instrumentation Engineering Work Bench). The TEEN is succeeded by the APTEEN, aims at capturing periodic data aggregations and respond to time-critical events (gives overall picture of the network periodically). APTEEN is a hybrid protocol for efficient routing and comprehensive information retrieval in WSN [3]. Is important to analyse energy required because of remote use of WSN. Also easier analysis of vibration with sound and vibration tools kit by National Instrument.

\section{Importance of WSN in oil and gas}

Oil and gas industry can use WSN to monitor underwater piping system vibrations (mechanical, big animals and deep soil structure impact). Vibro-acoustics is used to ensure integrity and operation reliability of pipelines in different scenarios for transport of fluids, design and calibrate advanced procedures for real-time pipeline monitoring [4]. The use of multi-point acoustic sensing at distance to detect third party interference (TPI) and validate mathematical models of pressure wave propagation in fluids filled pipes is currently industrialized [5]. Hazards significantly endanger pipeline function leading to damage, leakage and pipeline failure and each results in serious ecological and economic consequences [6].

\section{Aim and objectives}

The aim of the research is to develop a reliability model for pipelines by monitoring vibrations using WSN with energy network analysis. The objectives include; Model reliability for pipelines from vibrations template, low power consumption via energy network analysis tools, online monitoring of vibrations via LabVIEW user friendly interface with analysis by sound and vibration tools kit by National Instrument, to easily have fast data that can serve for future forecast/prediction maintenance, prevention and preparations (PPP) and the exact location of the pipeline using Global Positioning System (GPS). 


\section{Methodology}

The concept of monitoring pipelines can be executed by sensors in a WSN to detect different parameters on the pipeline which include acoustic sensor. The acoustic sensor will detect, monitor and analyse changes in the pipeline due to vibrations with aid of sound and vibration toolkit from National Instrument tools via LabVIEW user interface for timely action plan. Also to detect the presence of foreign object under water. Energy consumption is managed by protocols and network tools (matlab, netlogo) during data routing and clustering to ensure low power consumption by the nodes activity. Below in figure 1 . is the concept.

The acoustic technique will detect the vibration and the acoustic system will monitor from the pipeline or from the environment using an array of microphones networked underwater (WSN), send to a server via sensor node and conditioned and analyzed from an interface. Such vibrations can be categorized by sources and set a warning for each category of vibration source monitored (vibrations/frequency template from behavior of pipeline under conditions). A warning observed from monitoring a limit set for this vibration sources will allow an action plan on time.

The acoustic technique was employed to detect and account for the population of insects larvae under the soil [7]. The robot system was used to detect gas in the environment [8], this can serve to monitor (video/image or as a server) pipeline even under water with the help of acoustic system to detect changes in vibration and sound to avoid damage by foreign objects like animals, or on the surface for spillage with oil sensors.
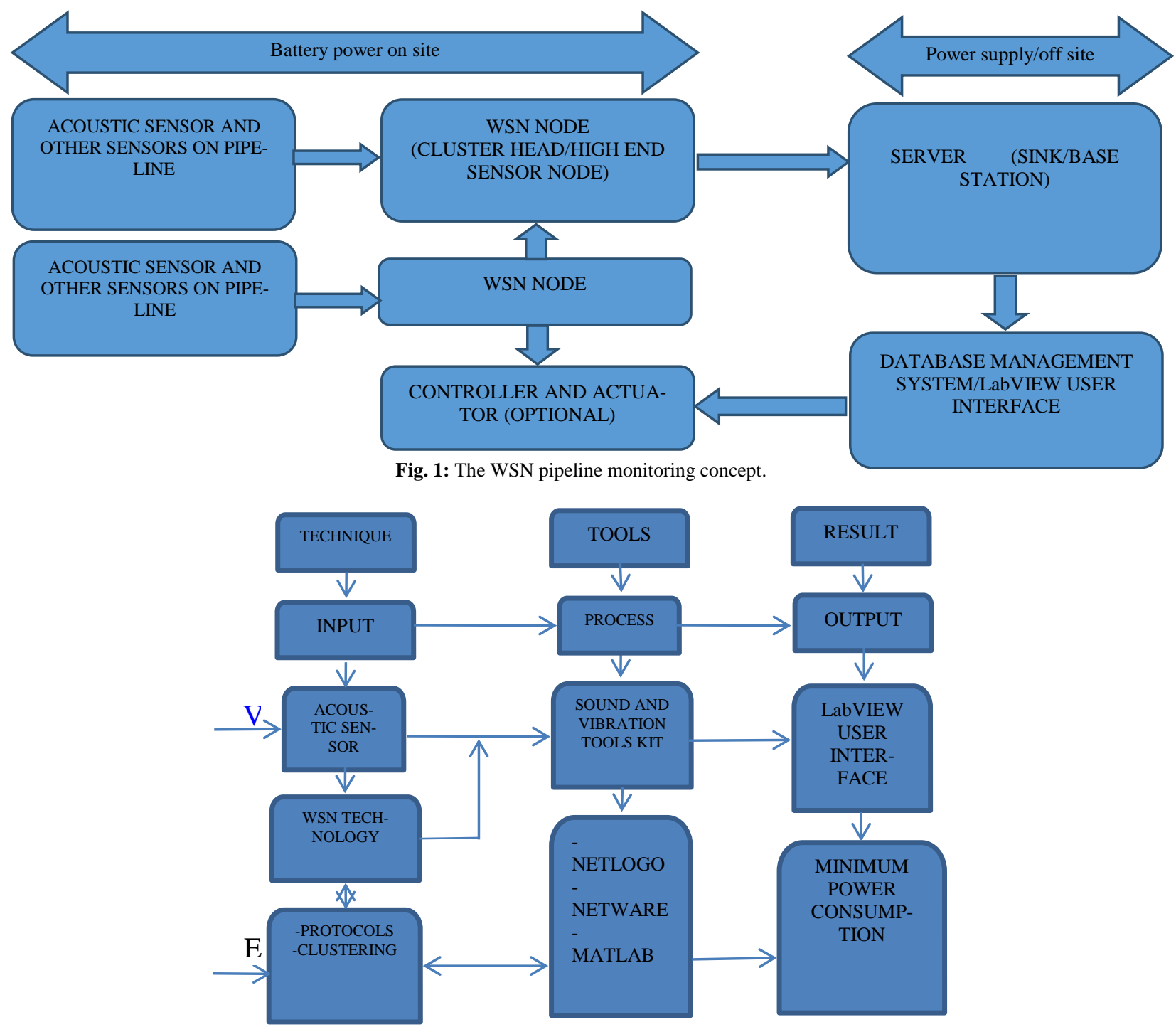

Fig. 2: Data mode block diagram.

\section{Mode of data collection}

Data collection starts with the sensors parameter detected, all sensors connected to a sensor node route data wirelessly to another node up cluster head (high end sensor node) and send to a computer via server/base station. The data from server is analyzed and viewed by sound and vibration tool kits via a friendly user interface (LabVIEW) on the computer. The WSN is a designed and analyzed to route data via protocols, clustering and network simulation tools to ensure low power consumption by nodes in network
Vibrations from mechanical parts, vandalization, earth-quake, natural phenomena (tidal, wind) and whales on the pipeline can be generated as template over time via LabVIEW with the sound and vibration tool kits. This template can be utilized to model a system reliability for the pipeline operation. Other sensor like liquefied petroleum gas (LPG) can detect spillage, pressure, temperature and flow sensors on the pipeline.

In the future, a Global Positioning System (GPS) for exact location of pipeline and a quad copter [9] or an underwater vehicle can 
be a server/base station. Also a universal sensor can be modeled for sensing parameters in harsh environment.

Data will be analyzed as shown in the diagram below in figure 2 . Data should be collected by experiment in lab then ocean (magnitude of parameters and conditions are scaled to be experimented in the laboratory). Reliability ensures low down time and increase production with sustainability.

\section{Conclusion}

This research best suits the oil and gas industry where the system is completely offshore or any pipeline system that is underwater. The reliability and integrity of such systems is paramount for optimum performance in which there is environmental constrains (harsh). Therefore, maintenance has to be effective (timely), this will provide predictive maintenance through reliability to enhance maintenance. This article provides a means/ methodology/ approach to monitor reliability of pipelines using acoustic system and detection via acoustic technique. The acoustic technique detects the vibration from the pipeline at different scenarios. Acoustic system using sound and vibration tools kit from National Instrument with LabVIEW interface. The vibration detection and monitoring with acoustic system is termed as vibro-acoustics. This will model the reliability of underwater pipeline. It has been implemented in the industries to monitor pipeline and their reliability. But in this case, we are to use it together with WSN to utilize the concept of low power consumption in WSN. The experiment will prove $90 \%$ of the hypothesis and improvement of the realtime result from monitoring with friendly user interface.

\section{Acknowledgement}

I acknowledge the contributions of my co-authors to put together the idea for the purpose of solving a problem and improvements in the aspect of solving the problem under my specialization.

\section{References}

[1] Qinghua Wang and Ilangko Balasingham. Wireless Sensor Networks - "An Introduction, Wireless Sensor Networks: ApplicationCentric Design”, Yen Kheng Tan (Ed.), ISBN: 978-953-307-321-7, (2010) InTech, Available from: http://www.intechopen.com/books/wireless-sensornetworksapplication-centricdesign/wireless-sensornetworks- anintroduction.

[2] Richard W. Mankin, Barukh Rohde, and Seth McNeill, "Vibrational duetting mimics to trap and disrupt mating of the devasatating Asian citrus psyllid insect pest," Proceedings of Meetings on Acoustics, Jacksonville, Florida, Vol. 25 010006, (2016)

[3] Rashid Hussain, Neha Sharma, "precision agriculture energy conservation in wireless sensor network (WSN) by using bioinspired quorum sensing method", (AESR 10), Volume-1, Issue-1, (2015).

[4] Giuseppe Giunta, Giancarlo Bernasconi, Silvio Del Giudice, "Pipeline Monitoring with Vibroacoustic Sensing”, Material Evaluation

[5] (ME Technical Paper), American Society of Non-destructive testing, Volume 73(7): (2015) 979-986.

[6] Giancarlo Bernasconi, Silvio Del Giudice, Giuseppe Giunta, Francesco Dionigi, "Advanced Pipeline Vibroacoustic Monitoring", American Society of Mechanical Engineers, pressure vessels and piping division (publication) PVP -97281(2013).

[7] Giuseppe Giunta, Silvia Morrea, Rennat Gabbassov, Giancarlo Bernasconi, Silvio Del Giudice, "Performance of Vibroacoustic Technology for Pipeline Leakage Detection", American Society of Mechanical Engineers, PVP Volume 5: pipelines, Risers and Subsea Systems (OMAE 2016-5421).

[8] Richard W. Mankin, Clayton W. McCoy, Kathy L. Flanders, Jamee Brandhorst, Robert L. Crocker, and Jeffrey P. Shapiro, "Methods for Acoustic Detection of Insect Pests in Soil," Proceedings of the Soil Science Society of America Conference on Agroacoustics, Third Symposium, Buoyoucos, MS 1998, (3/31/2016).
[9] V. M. Hernandez bennetts, " Mobile Robots with In-situ and remote sensors for real world gas distribution modeling," Orebro University, ISBN 978-91-7529-055-3, (12/2014), pp. 22-23.

[10] E. Puig, F. Gonzalez, G. Hamilton and P. Grundy, "Assessment of crop insect damage using unmanned aerial systems: A machine learning approach," 21st International Congress on Modelling and Simulation, Gold Coast, Australia, 29 Nov to 4 Dec 2015, www.mssanz.org.au/modsim 2015 